\title{
POTENCIAL DE Cajanus cajan E Crotalaria spectabilis PARA FITORREMEDIAÇÃO: ABSORÇÃO DE ARSÊNIO E RESPOSTAS ANTIOXIDATIVAS ${ }^{1}$
}

\author{
Rafaella Teles Arantes Felipe ${ }^{2}$, Juraci Alves de Oliveira ${ }^{3}$ e Gabriela Alves Leão²
}

\begin{abstract}
RESUMO - Neste estudo, avaliaram-se os efeitos tóxicos do arsênio (As) sobre o crescimento e atividade de enzimas antioxidativas dismutase do superóxido (SOD), catalase (CAT), peroxidases (POX), peroxidase do ascorbato peroxidase (APX) e glutationa redutase (GR) em plantas de Cajanus cajan e Crotalaria spectabili. Plantas das duas espécies foram expostas a diferentes concentrações de As, em solução nutritiva de Hoagland, pH 6,5. A taxa de crescimento relativo (TCR) das duas espécies reduziu-se com o aumento da concentração de As na solução nutritiva, sendo de forma mais intensa em $C$. cajan. A concentração de As na solução capaz de reduzir em $50 \%$ a TCR de C. cajan foi de $0,93 \mathrm{mg} \mathrm{L}^{-1}$, enquanto em C. spectabilis foi de $4,80 \mathrm{mg} \mathrm{L}^{-1}$. As duas espécies apresentaram reduzida translocação de As para a parte aérea, sendo em raízes de C. spectabilis observada a maior concentração desse elemento. C. cajan exposto ao As teve as atividades das enzimas CAT, POX e APX reduzidas, ao contrário do que ocorreu em C. spectabilis. Esta espécie, quando submetida ao As, apresentou incremento na atividade de todas as enzimas avaliadas. A maior tolerância ao As observada em C. spectabilis pode estar relacionada à maior capacidade em reter o As nas raízes e à indução das enzimas SOD, CAT, POX, APX e GR. Dessa forma, C. spectabilis é uma espécie com potencial para utilização em programas de revegetação de áreas contaminadas com As.
\end{abstract}

Palavras-chave: Fitoestabilização, revegetação e enzimas antioxidativas.

\section{POTENTIAL OF Cajanus cajan AND Crotalaria spectabilis FOR PHYTOREMEDIATION: ARSENIC ABSORPTION AND ANTIOXIDATIVE RESPONSES}

\begin{abstract}
The toxic effects of Arsenic (As) on the growth and activities of the antioxidative enzymes superoxide dismutase (SOD), catalase (CAT), peroxidase (POX), ascorbate peroxidase (APX) and glutathione reductase (GR) of Cajanus cajan and Crotalaria spectabilsi were studied. AQUI Plants of the two species were exposed to different As concentrations in Hoagland's nutrient solution, $p H$ 6.5. The relative growth rate (RGR) of the two species reduced with the increase in As concentration in the nutrient solution, especially in C. cajan. Arsenic concentration to reduce $50 \%$ in the RGR of C. cajan was $0.93 \mathrm{mg} \mathrm{L}^{-1}$ and for C. spectabilis 4.80 $m g L^{-1}$ of As. Both species showed reduced As translocation to the shoot and . spectabilis accumulated higher amount of As in the roots. The activities of the enzymes CAT, POX and APX decreasedd in C cajan, but increased in $\boldsymbol{C}$. spectabilis. The higher $\boldsymbol{C}$. spectabilis tolerance to As is probably due to its higher ability to retain As in the roots and to induce antioxidative enzymes. $\boldsymbol{C}$. spectabilis is therefore a better bioremediator than $\boldsymbol{C}$. cajan and it is potentially useful to be used in revegetation programs of As-polluted areas.
\end{abstract}

Keywords: Phytostabilization, revegetation and antioxidatives enzymes.

\footnotetext{
${ }^{1}$ Recebido em 14.09.2007 e aceito para publicação em 06.03.2009.

2 Programa de Pós-Graduação em Fisiologia Vegetal da Universidade Federal de Viçosa (UFV). E-mail: < rtfelipe@esalq.usp.br> e<gabrielaaalves@yahoo.com.br>.

${ }^{3}$ Departamento de Biologia Geral da UFV. E-mail: <jalves@ufv.br>.
} 


\section{INTRODUÇÃO}

A revegetação de solos degradados é prática crescente devido à necessidade de restabelecer ambientes que sofreram alterações por diversas intervenções, a por exemplo da atividade de mineração. Porém, algumas áreas apresentam concentrações elevadas de metais e metalóides tóxicos, o que agrava a situação desses locais por causa do risco de contaminação de solos vizinhos, águas superficiais e subterrâneas (BOULARBAH et al., 2006). Entre os diversos contaminantes oriundos de atividade de mineração, a liberação de arsênio (As) no ambiente é um dos fatores de maior risco para os ecossistemas, com sérios danos à saúde humana, que incluem efeitos mutagênicos, carcinogênicos e teratogênicos (TAPIO e GROSCHE, 2006).

A utilização de plantas na redução da contaminação do ambiente é uma alternativa passível de ser utilizada, em substituição ou complementação aos métodos físicoquímicos (ALDRICH et al., 2007). Para isso, há a necessidade de selecionar espécies vegetais capazes de tolerar as concentrações de elementos tóxicos nessas áreas, como o As presente em áreas de mineração.

$\mathrm{O}$ arsênio, na forma de arsenato $\left(\mathrm{As}^{5+}\right)$, predomina em ambientes aeróbicos e, devido à similaridade com o fosfato, pode participar de muitas reações celulares, como a substituição do fosfato em macromoléculas de DNA e a competição pelo transportador de fosfato, bem como inibir a síntese de ATP (PAIVOKE e SIMOLA, 2001; ROSEN, 2002). Uma vez absorvido, o arsenato pode ser reduzido a arsenito $\left(\mathrm{As}^{3+}\right)$ e inibir a atividade de várias enzimas (MEHARG e HARTLEY-WHITAKER, 2002) e, ou, induzir o aumento na produção de intermediários reativos de oxigênio (ROIs) (BHATTACHARYA e BHATTACHARYA, 2005), como o radical superóxido $\left(\mathrm{O}_{2}^{\cdot-}\right)$, o peróxido de hidrogênio $\left(\mathrm{H}_{2} \mathrm{O}_{2}\right)$ e o radical hidroxila $\left(\mathrm{OH}^{\bullet}\right)$.

As plantas exibem várias respostas bioquímicas para proteção contra agentes oxidantes, que incluem a ação de mecanismos enzimáticos, como aumento na atividade da dismutase do superóxido (SOD), da catalase (CAT), da peroxidase (POX), da peroxidase do ascorbato (APX) e da redutase da glutationa (GR).

Espécies vegetais fixadoras de nitrogênio, como as leguminosas Cajanus cajan (L.) Millsp e Crotalaria spectabilis Roth, têm sido indicadas para utilização em programas de revegetação de áreas degradadas, corroborado pelo fato de serem naturalmente encontradas nessas áreas (ARAÚJO et al., 2005). Entretanto, pouco se sabe sobre os mecanismos de tolerância dessas espécies quando expostas ao As (ZHANG et al., 2001; LINS et al., 2006). Em parte, essa indicação se deve à capacidade de essas espécies sobreviverem em ambientes degradados, com reduzida quantidade de matéria orgânica e de diversos nutrientes, principalmente de nitrogênio (WONG, 2003; SARCINELLI et al., 2004).

Os efeitos do As sobre sua absorção e distribuição, em espécies vegetais arbustivas indicadas para revegetação de áreas degradadas, são pouco estudados, uma vez que a maioria das pesquisas e realizada em ambientes degradados, mas sem a presença do As em níveis tóxicos. Dessa forma, essas informações são escassas para espécies indicadas para utilização em áreas de mineração contaminadas com As.

O objetivo deste estudo foi avaliar os efeitos tóxicos do As, na forma de $\mathrm{As}^{5+}$, no crescimento, na absorção e em sua distribuição nas plantas submetidas a diversas concentrações desse elemento. Análises da atividade das enzimas SOD, CAT, POX, APX e GR foram realizadas para evidenciar a existência de mecanismos de tolerância associados às espécies.

\section{MATERIAL E MÉTODOS}

\subsection{Obtenção das plantas}

Nos experimentos foram utilizadas sementes de C. cajan e C. spectabilis, fornecidas pelo Laboratório de Sementes do Departamento de Engenharia Florestal da Universidade Federal de Viçosa, em Viçosa, MG. Sementes de cada espécie foram colocadas para germinar em rolos de papel germtest, parcialmente imersos em recipientes contendo $1,5 \mathrm{~L}$ de água desionizada, em sala de crescimento, à temperatura de $25 \pm 2{ }^{\circ} \mathrm{C}$, no escuro. Quatro dias após a germinação, as plântulas foram selecionadas e transferidas para vasos (4 plantas/vaso) contendo $1,8 \mathrm{~L}$ da solução nutritiva de Clark (1975), com pH 6,5 ajustado diariamente, aeradas e colocadas em sala de crescimento, à temperatura de $25 \pm 2{ }^{\circ} \mathrm{C}$, sob irradiância de $230 \mu \mathrm{mol} \mathrm{m} \mathrm{m}^{-2} \mathrm{~s}^{-1}$ e fotoperíodo luminoso de $16 \mathrm{~h}$, no período de crescimento. 


\subsection{Experimento 1 - Efeito do As sobre a taxa de crescimento relativo (TCR) e determinação da concentração desse elemento nas plantas}

Decorridos oito dias de crescimento, as soluções foram substituídas, bem como foram aplicados os tratamentos com As, na forma de $\mathrm{Na}_{2} \mathrm{HAsO}_{4} \cdot 7 \mathrm{H}_{2} \mathrm{O}$, nas concentrações de 0,$0 ; 1,5 ; 2,5 ; 5,0 ; \mathrm{e} 7,5 \mathrm{mg} \mathrm{L}^{-1}$.

Após sete dias de exposição ao As, as plantas foram lavadas com água corrente e água desionizada, pesadas e separadas em raízes e parte aérea. A seguir, foram colocadas para secar em estufa convencional, à temperatura de $80^{\circ} \mathrm{C}$, até a obtenção do peso constante. $\mathrm{O}$ peso da massa seca inicial foi estimado a partir de amostras de plantas tomadas do mesmo lote das utilizadas no experimento.

As taxas de crescimento relativo (TCR) das plantas foram, então, calculadas utilizando-se a equação: TCR $=\left(\ln \mathrm{w}_{1}-\ln \mathrm{w}_{0}\right) \times 1000 /\left(\mathrm{t}_{1}-\mathrm{t}_{0}\right)$, em que TCR foi expressa em termos de ganho de massa seca, no período experimental $\left(\mathrm{mg} \mathrm{g}^{-1} \mathrm{dia}^{-1}\right)$, $\ln \mathrm{w}_{1}$ e $\ln \mathrm{w}_{0}$ correspondem ao logaritmo neperiano da massa seca no final e no início do experimento, respectivamente, e $\mathrm{t}_{1}-\mathrm{t}_{0}$ corresponde à duração do experimento, em dias (HUNT, 1978).

Amostras de $100 \mathrm{mg}$ de massa seca das plantas foram mineralizadas em mistura nitroperclórica 2:1 à temperatura de $90-100^{\circ} \mathrm{C}$, para evitar a volatilização do As, até a completa oxidação da matéria orgânica (Adaptado de ALDER e WILCOX, 1985). No extrato obtido, foi feita a determinação da concentração de As, utilizando-se espectrofotômetro de emissão óptica em plasma induzido, marca Perkin Elmer e modelo Optima $3300 \mathrm{DV}$.

\subsection{Experimento 2 - Efeito do As sobre as atividades}

Decorridos 35 dias de crescimento, necessários para a obtenção de massa fresca suficiente para as análises, as soluções foram substituídas, assim como foram aplicados os tratamentos com As na forma de $\mathrm{Na}_{2} \mathrm{HAsO}_{4} \cdot 7 \mathrm{H}_{2} \mathrm{O}$, nas concentrações de 0,0 e $1,5 \mathrm{mg} \mathrm{L}^{-1}$.

Após três dias de exposição ao As, as plantas foram lavadas com água corrente, desionizadas e separadas em raízes e folhas.

\subsubsection{Obtenção dos extratos enzimáticos brutos}

Os extratos enzimáticos brutos para a determinação das atividades da dismutase do superóxido (SOD, EC
1.15.1.1), da catalase (CAT, EC 1.11.1.6), das peroxidases (POX, EC 1.11.1.7), da peroxidase do ascorbato (APX, EC 1.11.1.11) e da redutase da glutationa (GR, EC 1.8.1.7) foram obtidos pela homogeneização de $0,3 \mathrm{~g}$ de folhas e de raízes em nitrogênio líquido, seguida de adição de $2 \mathrm{~mL}$ de meio de extração e de centrifugação a 12.000 $\mathrm{xg}$, por $15 \mathrm{~min}$, à temperatura de $4{ }^{\circ} \mathrm{C}$. O sobrenadante obtido foi utilizado como extrato bruto na determinação das atividades enzimáticas. Todas as etapas necessárias ao processo de extração foram executadas na temperatura de $4{ }^{\circ} \mathrm{C}$.

Os meios de extração foram: 1) tampão fosfato de potássio 0,1 mol $\mathrm{L}^{-1}, \mathrm{pH}$ 6,8, ácido etilenodiaminotetracético (EDTA) $0,1 \mathrm{mmol} \mathrm{L}^{-1}$, fluoreto de fenilmetilsulfônico (PMSF) $1 \mathrm{mmol} \mathrm{L}^{-1} \mathrm{e}$ polivinilpirrolidona (PVPP) 1\% (p/v) (PEIXOTO et al., 1999), para as enzimas SOD, CAT e POX; 2) tampão fosfato de potássio $50 \mathrm{mmol} \mathrm{L}^{-1}, \mathrm{pH} 7,0$, EDTA $1 \mathrm{mmol}$ $\mathrm{L}^{-1}$, ácido ascórbico $1 \mathrm{mmol} \mathrm{L}^{-1}, \mathrm{PMSF} 1 \mathrm{mmol} \mathrm{L}^{-1}$, ditiotreitol (DTT) $2 \mathrm{mmol} \mathrm{L}^{-1}$ e PVPP 1\% (p/v) (PEIXOTO et al., 1999), para a APX; 3 ) tampão fosfato de potássio 0,1 mol L ${ }^{-1}, \mathrm{pH} 7,0$, EDTA $1 \mathrm{mmol} \mathrm{L}^{-1}$, triton 0,02\% (v/v), DTT 2 mmol L ${ }^{-1}$, PMSF $1 \mathrm{mmol} \mathrm{L}^{-1}$ e PVPP $1 \%(\mathrm{p} / \mathrm{v})$ (CARLBERG e MANNERVIK, 1985), para a GR.

\subsubsection{Determinação da atividade da SOD}

A atividade da SOD foi determinada pela adição de $50 \mu \mathrm{L}$ do extrato enzimático bruto a $5 \mathrm{~mL}$ de meio de reação constituído de tampão-fosfato de sódio 50 mmol L-1, pH 7,8, contendo metionina $13 \mathrm{mmol} \mathrm{L}^{-1}$, azul de p-nitro tetrazólio (NBT) $75 \mu \mathrm{mol} \mathrm{L}^{-1}$, EDTA 0,1 mmol $\mathrm{L}^{-1}$ e riboflavina $2 \mu \mathrm{mol} \mathrm{L} \mathrm{L}^{-1}$. A reação foi conduzida a $25{ }^{\circ} \mathrm{C}$, numa câmara de reação, sob iluminação de lâmpada fluorescente de $15 \mathrm{~W}$, mantida no interior de uma caixa coberta com papel-alumínio. Após 5 min de exposição à luz, a iluminação foi interrompida, e a formazana azul produzida pela fotorredução do NBT foi medida pela absorvância a $560 \mathrm{~nm}$. A absorvância a $560 \mathrm{~nm}$ de um meio de reação exatamente igual ao anterior, mas mantido no escuro por igual período, serviu de branco e foi subtraído da leitura da amostra que recebeu iluminação (GIANNOPOLITIS e RIES, 1977). Uma unidade de SOD foi definida como a quantidade de enzima necessária para inibir em $50 \%$ a fotorredução do NBT (BEAUCHAMP e FRIDOVICH, 1971).

\subsubsection{Determinação da atividade da CAT}

A atividade da CAT foi determinada pela adição de $0,1 \mathrm{~mL}$ do extrato enzimático bruto a $2,9 \mathrm{~mL}$ de meio

R. Árvore, Viçosa-MG, v.33, n.2, p.245-254, 2009 
de reação constituído de tampão fosfato de potássio 50 mmol L ${ }^{-1}, \mathrm{pH}_{7}, 0 \mathrm{eH}_{2} \mathrm{O}_{2}$ 12,5 $\mathrm{mmol} \mathrm{L}^{-1}$ (HAVIR e MCHALE, 1987). O decréscimo na absorvância, no primeiro minuto de reação, foi medidoa $240 \mathrm{~nm}$, a $25^{\circ} \mathrm{C}$. Aatividade enzimática foi calculada utilizando-se o coeficiente de extinção molar de $36 \mathrm{~mol}^{-1} \mathrm{~L} \mathrm{~cm}^{-1}$ (ANDERSON et al., 1995) e expressa em $\mu \mathrm{mol}$ de $\mathrm{H}_{2} \mathrm{O}_{2} \min ^{-1} \mathrm{~g}^{-1} \mathrm{MF}$.

\subsubsection{Determinação da atividade das POX}

A atividade das POX foi determinada pela adição de $0,1 \mathrm{~mL}$ do extrato enzimático bruto a $4,9 \mathrm{~mL}$ de meio de reação constituído de tampão fosfato de potássio $25 \mathrm{mmol} \mathrm{L}^{-1}$, pH 6,8, pirogalol $20 \mathrm{mmol} \mathrm{L}^{-1}$ e $\mathrm{H}_{2} \mathrm{O}_{2} 20$ mmol L-1 (KAR e MISHRA, 1976). A produção de purpurogalina foi determinada pelo incremento da absorvância durante o primeiro minuto de reação a $420 \mathrm{~nm}$, a $25^{\circ} \mathrm{C}$. A atividade enzimática foi calculada utilizando-se o coeficiente de extinção molar de 2,47 $\mathrm{mmol}^{-1} \mathrm{Lcm}^{-1}$ (CHANCE e MAEHLEY, 1955) e expressa em $\mu \mathrm{mol}$ de $\mathrm{H}_{2} \mathrm{O}_{2} \min ^{-1} \mathrm{~g}^{-1} \mathrm{MF}$.

\subsubsection{Determinação da atividade da APX}

A atividade da APX foi determinada pela adição de $0,1 \mathrm{~mL}$ do extrato enzimático bruto a $2,9 \mathrm{~mL}$ de meio de reação constituído de tampão fosfato de potássio $50 \mathrm{mmol}$ $\mathrm{L}^{-1}, \mathrm{pH}$ 6,0, ácido ascórbico $0,8 \mathrm{mmol} \mathrm{L}^{-1} \mathrm{e} \mathrm{H}_{2} \mathrm{O}_{2} 1 \mathrm{mmol}$ $\mathrm{L}^{-1}$ (NAKANOeASADA, 1981, modificado por KOSHIBA, 1993). O decréscimo na absorvância a $290 \mathrm{~nm}$ foi medido no primeiro minuto de reação, a $25^{\circ} \mathrm{C}$. A atividade enzimática foi calculada utilizando-se o coeficiente de extinção molar de $2,8 \mathrm{mmol}^{-1} \mathrm{Lcm}^{-1}$ (NAKANOeASADA, 1981) e expressa em $\mu$ mol de ácido ascórbico $\mathrm{min}^{-1} \mathrm{~g}^{-1} \mathrm{MF}$.

\subsubsection{Determinação da atividade da GR}

A atividade da GR foi determinada pela adição de $0,1 \mathrm{~mL}$ do extrato enzimático bruto a $0,9 \mathrm{~mL}$ de um meio de reação constituído de tampão tris- $\mathrm{HCl} 0,1 \mathrm{~mol}$ $\mathrm{L}^{-1}, \mathrm{pH} 7,5$, GSSG $1 \mathrm{mmol} \mathrm{L}^{-1}$ e NADPH 0,1 $\mathrm{mmol} \mathrm{L}^{-1}$ (CARLBERG e MANNERVIK, 1985). O decréscimo na absorvância a $340 \mathrm{~nm}$ foi medido no primeiro minuto de reação, a $30^{\circ} \mathrm{C}$. A atividade enzimática foi calculada utilizando-se o coeficiente de extinção molar de 6,22 $\mathrm{mmol}^{-1} \mathrm{~L} \mathrm{~cm}^{-1}$ e expressa em $\mu \mathrm{mol}$ de GSSG min ${ }^{-1} \mathrm{~g}^{-1}$ MF.

\subsection{Delineamento experimental e análise estatística}

O experimento para determinação da taxa de crescimento relativo e acúmulo de As foi conduzido no esquema fatorial 2 × 5 (duas espécies vegetais e cinco concentrações de As), no delineamento em blocos casualizados, com três repetições. O experimento para avaliação das atividades enzimáticas foi conduzido no esquema fatorial 2 × 2 (duas espécies vegetais e duas concentrações de As), no delineamento inteiramente casualizado, com três repetições. As variáveis que não atenderam aos pressupostos para realização da análise de variância (normalidade e homocedásticidade) foram transformadas: os dados das variáveis "concentração de As em raízes" foram transformados em $\log (x+50)$, "POX de raízes" e "GR de folhas" transformados em $\sqrt{x+0,5} \mathrm{e}$ "APX de folhas" transformados em $\log (\mathrm{x})$. Atendidos os pressupostos, os dados foram, então, submetidos à análise de variância e as médias comparadas pelo teste de Scott-Knot a 5\% de probabilidade.

\section{RESULTADOS}

\subsection{Concentração de As nas plantas e efeitos sobre a taxa de crescimento relativo}

As TCR das espécies C. cajan e C. spectabilis mostraram-se semelhantes em condições de cultivo sem a adição de As. Entretanto, quando expostas a concentrações crescentes de As, as TCR C. spectabilis foram muito superiores àquelas apresentadas pela espécie C. cajan (Tabela 1).

Aexposição das plantas ao As provocou significativa redução na TCR nas duas espécies, sendo de forma mais intensa em C. cajan. Ficou evidente a maior severidade do dano em $C$. cajan, em que a concentração de As, em solução, capaz de causar redução de 50\% na TCR foi de $0,93 \mathrm{mg} \mathrm{L}^{-1}$, enquanto em C. spectabilis foi de 4,80 $\mathrm{mg} \mathrm{L}^{-1}$ (Figura 1).

Tabela 1 - Taxa de crescimento relativo de $C$. cajan e $C$. spectabilis submetidas a diferentes concentrações de As, em solução nutritiva, durante sete dias

Table 1 -Relative growth rate of C. cajan e C. spectabilis submitted to different As concentrations, in nutritive solution, for seven days

\begin{tabular}{ccc}
\hline Concentração & \multicolumn{2}{c}{ TCR $\left(\mathrm{mg} \mathrm{dia}^{-1} \mathrm{~g}^{-1} \mathrm{MS}\right)$} \\
\cline { 2 - 3 } de As $\left(\mathrm{mg} \mathrm{L}^{-1}\right)$ & C. cajan & C. spectabilis \\
\hline 0,0 & $127,20 \mathrm{a}$ & $129,49 \mathrm{a}$ \\
1,5 & $53,44 \mathrm{~b}$ & $103,29 \mathrm{a}$ \\
2,5 & $29,59 \mathrm{~b}$ & $86,79 \mathrm{a}$ \\
5,0 & $7,53 \mathrm{~b}$ & $64,75 \mathrm{a}$ \\
7,5 & $-12,57 \mathrm{~b}$ & $26,40 \mathrm{a}$ \\
\hline
\end{tabular}

As médias seguidas pelas mesmas letras nas linhas não diferem estatisticamente entre si, pelo teste Scott-Knot a $5 \%$ de probabilidade. 

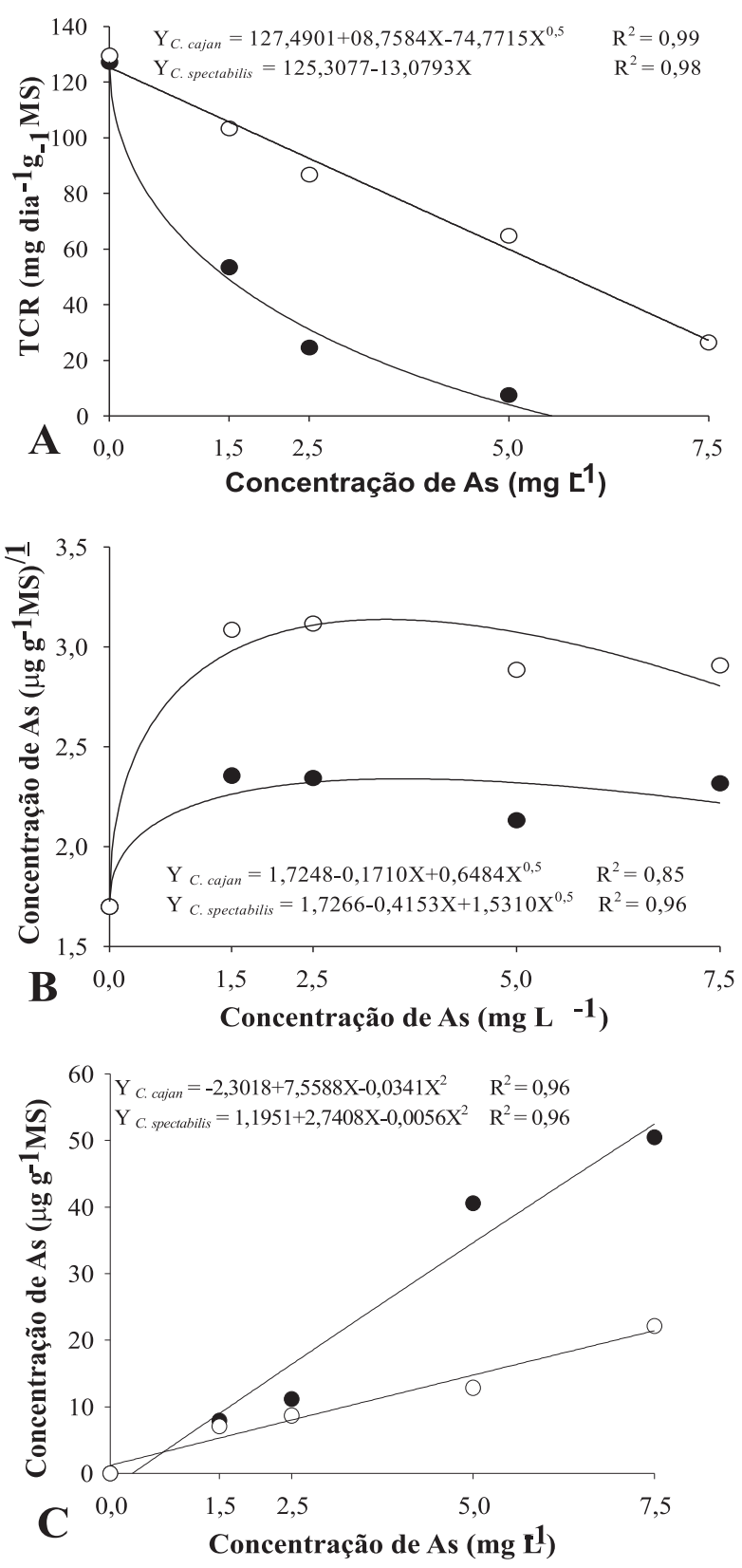

1/ Dados transformados em $\log (\mathrm{x}+50)$.

Figura 1 - Taxa de crescimento relativo (A), concentração de As em raízes (B) e parte aérea (C) de C. cajan $(\bullet)$ e C. spectabilis $(\bigcirc)$ submetidas a diferentes concentrações de As, em solução nutritiva, durante sete dias.

Figure 1 - Relative growth rate $(A)$, concentration in the roots $(B)$ and in the shoot $(C)$ of $C$. cajan $(\bullet) e$ C. spectabilis (O) submitted to different As concentrations, in nutritive solution, for seven days.
A elevada sensibilidade da espécie $C$. cajan ao As ficou evidenciada, também, quando foi avaliada a concentração desse elemento nas plantas capaz de causar a redução de 50\% na TCR, sendo de 113,7 $\mu \mathrm{g}$ $\mathrm{g}^{-1} \mathrm{MS}$ de As em C. cajan e de $1.164,26 \mu \mathrm{g} \mathrm{g}^{-1} \mathrm{MS}$ de As, em C. spectabilis.

A espécie $C$. spectabilis apresentou maiores concentrações de As nas raízes do que $C$. cajan, com reduzida translocação para a parte aérea. Entretanto, na parte aérea, apesar da pequena translocação, a espécie C. cajan teve concentrações maiores do que $C$. spectabilis, a partir da concentração de $2,5 \mathrm{mg} \mathrm{L}^{-1}$ (Tabela 2).

A concentração de As nas raízes das duas espécies aumentou até a concentração de $2,5 \mathrm{mg} \mathrm{L}^{-1}$ de As em solução, seguida de decréscimo nas maiores concentrações. C. cajan apresentou máxima concentração de As em suas raízes, 149,52 $\mu \mathrm{g} \mathrm{g}^{-1} \mathrm{MS}$, quando submetida a 3,6 mg $\mathrm{L}^{-1}$ de As, e $C$. spectabilis apresentou máxima concentração de As em suas raízes, $1.208,92 \mu \mathrm{g} \mathrm{g}^{-1} \mathrm{MS}$, quando submetida a 3,4 $\mathrm{mg} \mathrm{L}^{-1}$ desse elemento (Figura 1).

$\mathrm{Na}$ parte aérea, C. cajan e C. spectabilis apresentaram incremento na concentração de As com o aumento da concentração deste elemento em solução, sendo de forma mais intensa em $C$. cajan (Figura 1).

\subsection{Efeitos do As e respostas de enzimas antioxidativas}

A atividade da SOD, em folhas, foi maior na espécie C. spectabilis em todos os tratamentos e, mesmo quando exposta ao As, não sofreu alteração (Tabela 3 ).

Quando cultivada em solução sem As, a espécie C. cajan teve maior atividade da SOD em raízes do que C. spectabilis. Entretanto, sob exposição ao As, observou-se incremento de $70 \%$ na atividade da enzima em $C$. spectabilis, enquanto em $C$. cajan a atividade foi mantida sem alteração significativa (Tabela 3 ).

A atividade da CAT em folhas de C. cajan foi maior do que em C. spectabilis, independentemente da concentração de As. Em raízes, entretanto, a exposição ao As ocasionou redução de $34 \%$ na atividade da CAT em $C$. cajan, enquanto em $C$. spectabilis houve incremento de $116 \%$ (Tabela 3 ).

A atividade das POX foi maior em folhas de $C$. cajan e não se observou alteração significativa quando as plantas, das duas espécies, foram expostas ao As. Em raízes, entretanto, a exposição ao As causou redução na atividade da peroxidase em $C$. cajan e aumento na atividade dessa enzima em $C$. spectabilis (Tabela 3 ). 
Tabela 2 - Concentração de As nas raízes e parte aérea de $C$. cajan e $C$. spectabilis submetidas a diferentes concentrações de As, em solução nutritiva, durante sete dias

Table 2 - As concentration in the roots and in the shoot of C. cajan e C. spectabilis submitted to different As concentrations, in nutritive solution, for seven days

\begin{tabular}{|c|c|c|c|c|}
\hline \multirow[t]{3}{*}{ Concentração de As $\left(\mathrm{mg} \mathrm{L}^{-1}\right)$} & \multicolumn{4}{|c|}{ Concentração As $\left(\mu \mathrm{g} \mathrm{g}^{-1} \mathrm{MS}\right)$} \\
\hline & \multicolumn{2}{|c|}{ Raiz } & \multicolumn{2}{|c|}{ Parte aérea } \\
\hline & C. cajan & C. spectabilis & C. cajan & C. spectabilis \\
\hline 0,0 & $0,00 \mathrm{a}$ & $0,00 \mathrm{a}$ & 0,0 a & $0,0 \mathrm{a}$ \\
\hline 1,5 & $177,12 \mathrm{~b}$ & 1169,33 a & 7,94 a & 7,03 a \\
\hline 2,5 & $170,58 \mathrm{~b}$ & $1273,05 \mathrm{a}$ & $11,18 \mathrm{a}$ & $8,67 \mathrm{~b}$ \\
\hline 5,0 & $85,83 \mathrm{~b}$ & 716,52 a & $40,57 \mathrm{a}$ & $12,84 \mathrm{~b}$ \\
\hline 7,5 & $157,89 \mathrm{~b}$ & 763,68 a & 50,45 a & $22,14 \mathrm{~b}$ \\
\hline
\end{tabular}

Média dos dados originais: as médias seguidas pelas mesmas letras nas linhas não diferem estatisticamente entre si, pelo teste de ScottKnot a 5\% de probabilidade. Para efeito de análise estatística, os dados da variável "concentração de As nas raízes" foram transformados em $\log (\mathrm{x}+50)$.

Tabela 3 - Atividade da SOD, CAT, POX, APX e GR em folhas e raízes de C. cajan e C. spectabilis submetidas a 0,0 e $1,5 \mathrm{mg} \mathrm{L}^{-1}$ de As, em solução nutritiva, durante três dias

Table 3 - SOD, CAT, POX, APX e GR activity in leaves and in roots of C. cajan e C. spectabilis submitted to 0,0 e 1,5 $m g L^{-1}$ of $A s$, in nutritive solution, for three days

\begin{tabular}{|c|c|c|c|c|c|c|}
\hline \multirow[t]{3}{*}{$\overline{\text { Concentração de As }\left(\mathrm{mg} \mathrm{L}^{-1}\right)}$} & \multicolumn{6}{|c|}{ Compartimento } \\
\hline & \multicolumn{3}{|c|}{ Folhas } & \multicolumn{3}{|c|}{ Raízes } \\
\hline & C. cajan & C. spectabilis & Média & C. cajan & C. spectabilis & Média \\
\hline & \multicolumn{6}{|c|}{ Atividade da SOD (Unidades de SOD g ${ }^{-1}$ MF) } \\
\hline 0,0 & $9,38 \mathrm{Ab}$ & $11,50 \mathrm{Aa}$ & 10,44 & $14,02 \mathrm{Aa}$ & $9,41 \mathrm{Bb}$ & 11,72 \\
\hline 1,5 & $3,87 \mathrm{Ab}$ & $10,32 \mathrm{Aa}$ & 7,01 & $11,98 \mathrm{Ab}$ & $16,18 \mathrm{Aa}$ & 14,08 \\
\hline Média & 6,63 & 10,91 & 8,73 & 13,00 & 12,80 & 12,9 \\
\hline & \multicolumn{6}{|c|}{ Atividade da CAT $\left(\mu \mathrm{mol}\right.$ de $\left.\mathrm{H}_{2} \mathrm{O}_{2} \min ^{-1} \mathrm{~g}^{-1} \mathrm{MF}\right)$} \\
\hline 0,0 & 1841,66 & 545,43 & $1193,55 \mathrm{~B}$ & $174,15 \mathrm{Aa}$ & $60,16 \mathrm{Bb}$ & 117,16 \\
\hline 1,5 & 2171,98 & 685,26 & $1428,62 \mathrm{~A}$ & $114,97 \mathrm{Ba}$ & $131,88 \mathrm{Aa}$ & 123,43 \\
\hline Média & 2006,82 a & $615,35 \mathrm{~b}$ & 1311,08 & 144,56 & 96,02 & 120,29 \\
\hline \multicolumn{7}{|c|}{ Atividade da POX $\left(\mu \mathrm{mol}\right.$ de $\left.\mathrm{H}_{2} \mathrm{O}_{2} \mathrm{~min}^{-1} \mathrm{~g}^{-1} \mathrm{MF}\right)$} \\
\hline 0,0 & 247,80 & 86,03 & 166,91 & $688,50 \mathrm{Aa}$ & $698,84 \mathrm{Ba}$ & 693,67 \\
\hline 1,5 & 272,87 & 108,72 & 190,79 & $485,71 \mathrm{Bb}$ & $804,40 \mathrm{Aa}$ & 645,06 \\
\hline Média & $260,33 \mathrm{a}$ & $97,37 \mathrm{~b}$ & 178,85 & 587,11 & 751,62 & 669,36 \\
\hline & \multicolumn{6}{|c|}{ Atividade da APX ( $\mu \mathrm{mol}$ de ascorbato $\mathrm{min}^{-1} \mathrm{~g}^{-1} \mathrm{MF}$ ) } \\
\hline 0,0 & 20,88 & 6,18 & $13,53 \mathrm{~B}$ & $8,46 \mathrm{Aa}$ & $1,19 \mathrm{Bb}$ & 4,82 \\
\hline 1,5 & 26,95 & 7,51 & $17,23 \mathrm{~A}$ & $0,94 \mathrm{Bb}$ & $3,65 \mathrm{Aa}$ & 2,29 \\
\hline Média & $23,91 \mathrm{a}$ & $6,84 \mathrm{~b}$ & 15,38 & 4,70 & 2,42 & 3,56 \\
\hline \multicolumn{7}{|c|}{ Atividade da GR ( $\mu \mathrm{mol}$ de glutationa oxidada $\min ^{-1} \mathrm{~g}^{-1} \mathrm{MF}$ ) } \\
\hline 0,0 & 5,53 & 2,51 & $4,02 \mathrm{~B}$ & 0,88 & 0,95 & $0,91 \mathrm{~B}$ \\
\hline 1,5 & 7,27 & 3,71 & $5,49 \mathrm{~A}$ & 1,87 & 2,63 & $2,25 \mathrm{~A}$ \\
\hline Média & $6,40 \mathrm{a}$ & $3,11 \mathrm{~b}$ & 4,76 & 1,37 & 1,79 & 1,58 \\
\hline
\end{tabular}

Média dos dados originais: médias seguidas pelas mesmas letras minúsculas nas linhas e maiúsculas nas colunas não diferem significativamente entre si, pelo teste de Scott-Knot a 5\% de probabilidade. Para efeito de análise estatística, os dados das variáveis "POX de raízes" e "GR de folhas" foram transformados em $\sqrt{x+0,5}$ e "APX de folhas" em $\log (\mathrm{x})$.

Em folhas da espécie C. cajan, a atividade da APX foi maior do que em C. spectabilis, independentemente da concentração de As. Maiores atividades foram, também, observadas em raízes das plantas cultivadas em solução sem As. Entretanto, quando C. cajan foi exposta ao As, essa atividade se reduziu intensamente, assim como ocorreu na atividade da CAT e da POX.
Em C. spectabilis, assim como ocorreu com as atividades da SOD, da CAT e da POX, a exposição ao As resultou em aumento na atividade da APX, superando os valores apresentados por C. cajan (Tabela 3 ).

A atividade da GR, em folhas, foi maior em C. cajan, independentemente da concentração de As. Em raízes, com a exposição das plantas ao As, verificou-se 
incremento na atividade da GR em ambas as espécies (Tabela 3).

\section{DISCUSSÃO}

A menor ação tóxica do As na espécie $C$. spectabilis foi demonstrada pela menor redução na sua TCR, mesmo sob elevadas concentrações desse elemento em seus tecidos. Isso indica a maior tolerância dessa espécie ao As e seu potencial para utilização em revegetação de áreas contaminadas com esse elemento, uma vez que a tolerância é uma das principais características para seleção de espécies potencialmente úteis para revegetação de áreas contaminadas (SAHI et al., 2007).

A maioria das espécies vegetais estudadas retém o As absorvido, predominantemente, em suas raízes, como observado nas espécies $C$. cajan e $C$. spectabilis. Isso pode ocorrer devido à capacidade de compartimentação do As, principalmente no vacúolo dos tecidos radiculares, resultando em menor translocação para a parte aérea (CARBONELL-BARRACHINA et al., 1997) e, consequentemente, redução dos efeitos tóxicos do As. Isso pode estar relacionado à maior tolerância apresentada por C. spectabilis ao As, com retenção de $97 \%$ do As absorvido em suas raízes, enquanto $C$. cajan reteve cerca de $75 \%$. Adicionalmente, a maior retenção do elemento absorvido nas raízes é de grande importância em programas de revegetação de áreas contaminadas, pois diminui o risco de dispersão do contaminante no ambiente (BOULARBAH et al., 2006; MADEJÓN e LEPP, 2007) e sua entrada na cadeia alimentar (FRENCH et al., 2006).

C. cajan demonstrou elevada sensibilidade ao As, com intensa redução na TCR, mesmo acumulando menores concentrações de As. Na concentração de $7,5 \mathrm{mg} \mathrm{L}^{-1} \mathrm{de} \mathrm{As}$, as plantas de C. cajan morreram ao final do experimento, resultando em perda de massa seca e conseqüente obtenção de valor negativo de TCR. A redução na produção de biomassa pode ocorrer por restrições no transporte radicular, prejudicando a absorção de água e nutrientes (CARBONELLBARRACHINA et al., 1997) ou, devido ao aumento na produção de ROIs, causando vários danos celulares, como a peroxidação de lipídeos (BHATTACHARYA e BHATTACHARYA, 2005). A atuação de enzimas antioxidativas, como a dismutase do superóxido (SOD), a catalase (CAT), as peroxidases (POX), a peroxidase do ascorbato (APX) e a redutase da glutationa (GR) podem evitar a ação tóxica dos ROIs e, assim, conferir maior tolerância a esse elemento tóxico (CAO et al., 2004; SANTOS, 2006).

A atividade da enzima SOD na espécie C. cajan, aparentemente, não foi capaz de atenuar os danos causados pelo aumento do $\mathrm{O}_{2}{ }^{-}$e, consequentemente, pode ter levado à produção de outros ROIs, aumentando os danos oxidativos celulares. Verificou-se que, para outros elementos de menor potencial tóxico, essa redução ocorreu apenas em elevadas concentrações do elemento, conforme observado na espécie $C$. cajan submetida a diferentes concentrações de Zn e Ni (MADHAVA RAO e SRESTY, 2000).

Estudos com Zea mays (MYLONA et al., 1998), Trifolium pratense (MASCHER et al., 2002), Pteris vittata e Pteris ensiforms (SINGH et al., 2006), Azolla caroliniana, Lemna gibba e Salvinia minima (SANTOS, 2006) mostraram aumento na atividade da SOD após exposição ao As. Neste trabalho, esse aumento foi observado apenas em raízes de $C$. spectabilis, confirmando a importância dessa enzima como parte do mecanismo de defesa antioxidante, o que confere a maior tolerância ao As.

Os aumentos na atividade da CAT em folhas de C. cajan e em folhas e em raízes de C. spectabilis, tratadas com As, indicam que houve incremento na produção de $\mathrm{H}_{2} \mathrm{O}_{2}$, conforme observado em condições de estresse oxidativo (MITTLER, 2002), mas que esse aumento de $\mathrm{H}_{2} \mathrm{O}_{2}$ não resultou em efeitos tóxicos, devido à ação antioxidante dessa enzima. Estudos com Azolla caroliniana, Lemna gibba e Salvinia minima, submetidas a cinco diferentes concentrações de As, mostraram aumento na atividade da CAT nas menores concentrações e redução na atividade dessa enzima, nas maiores concentrações de As (SANTOS, 2006). Essa redução foi observada em raízes de $C$. cajan, evidenciandose que o excesso de ROIs possa ter provocado a oxidação da CAT (RONSEIN et al., 2006).

Algumas espécies vegetais, como o tomate, quando expostas ao As apresentam redução na atividade das POX (MITEVA et al., 2005), assim como observado em raízes de $C$. cajan, neste trabalho. Em outras espécies, no entanto, observa-se incremento na atividade dessa enzima (MASCHER et al., 2002; CAO et al., 2004), assim como observado em raízes de C. spectabilis, confirmando que, em algumas espécies, as POX fazem parte do mecanismo de defesa antioxidante contra os danos oxidativos provocados pelo As.

R. Árvore, Viçosa-MG, v.33, n.2, p.245-254, 2009 
Estudos indicam que a atividade da APX é importante componente do mecanismo antioxidante quando as espécies vegetais são expostas a concentrações menores de As (CAO et al., 2004; SANTOS, 2006), mas, quando expostas às maiores concentrações, ocorre significativa redução na sua eficiência e, ou, na sua síntese (RONSEIN et al., 2006). Possivelmente, isso pode ter ocorrido em C. cajan, que, mesmo apresentando maior atividade da enzima em folhas e em raízes, teve expressiva redução na atividade dessa enzima em raízes, quando submetida ao As. Na espécie C. spectabilis exposta ao As, por sua vez, observouse o incremento na atividade dessa enzima em folhas e em raízes, demonstrando que essa enzima, juntamente com as demais POX, a SOD e aCAT são de grande importância nas respostas de defesa contra o estresse oxidativo provocado pelo As, nessa espécie.

O incremento na atividade da enzima GR é importante componente do mecanismo antioxidante das plantas expostas a elementos tóxicos, conforme observado em Sesbania drummondii exposta ao Hg (ISRAR et al., 2006) e Azolla caroliniana, Lemna gibba e Salvinia mínima, expostas ao As (SANTOS, 2006). Neste trabalho, o aumento na atividade da GR, em folhas e raízes das duas espécies, reforça a participação dessa enzima como agente de proteção antioxidativa, através do aumento na razão glutationa reduzida/glutationa oxidada(GSH/GSSG). AGSH, por sua vez, além de fazer parte do ciclo ascorbato/glutationa, é capaz de eliminar alguns ROIs.

As duas espécies avaliadas apresentaram comportamentos distintos com relação às atividades enzimáticas. $C$. spectabilis mostrou incremento na atividade de todas as enzimas analisadas (SOD, CAT, POX, APX e GR), principalmente em suas raízes, onde foi determinada a maior concentração do As absorvido. Já C. cajan apresentou redução na atividade da CAT, POX eAPX, principalmente em suas raízes, onde também foi observada a maior concentração do As absorvido.

Dessa forma, o crescimento de plantas de $C$. spectabilis, apesar de apresentar maior acúmulo de As, foi menos afetado do que $C$. cajan, indicando maior tolerância ao As. O menor efeito tóxico do As em plantas de C. spectabilis está relacionado, em parte, à atuação dos mecanismos enzimáticos antioxidativos e, apesar de outros estudos serem necessários para conhecer melhor esses e outros mecanismos de tolerância ao As, C. spectabilis apresenta-se com promissoras possibilidades de utilização em programas de revegetação de áreas contaminadas com As.

R. Árvore, Viçosa-MG, v.33, n.2, p.245-254, 2009

\section{AGRADECIMENTOS}

Ao CNPq e à CAPES, pelas bolsas de pesquisa; e à FAPEMIG, pelo financiamento do projeto.

\section{REFERÊNCIAS}

ALDER, P. R.; WILCOX, G. E. Rapid perchloric acid digest methods for analysis of major elements in plants tissue. Communications in Soil Science and Plant Analysis, v.16, NUMERO, p. 1153-1163, 1985.

ALDRICH, M. V. et al. Examination of arsenic(III) and (V) uptake by the desert plant species mesquite (Prosopis spp.) using X-ray absorption spectroscopy. Science of the Total Environment, v.379, n.2, p.249-255, 2007.

ANDERSON, M. D.; PRASAD, T. K.; STEWART, C. R. Changes in isozyme profiles of catalase, peroxidase, and glutathione reductase during acclimation to chilling in mesocotylus of maize seedlings. Plant Physiology, v. 109, n.4, p.1247-1257, 1995.

ARAÚJO, F. S. et al. Florística da vegetação arbustivo-arbórea colonizadora de uma área degradada por mineração de caulim, em Brás Pires, MG. Revista Árvore, v.29, n.6, p.107-116, 2005.

BEAUCHAMP, C.; FRIDOVICH, I. Superoxide dismutase: improved assays and assay aplicable to acrylamide gels. Analytical

Biochemistry, v.44, n.1, p.276-287, 1971.

BHATTACHARYA, A. \& BHATTACHARYA, S. Indution of stress by arsenic in Clarus batrachus: Involvemente of peroxisomes. Ecotoxicology and Environmental Safety, v.66, n.2, p.178-187, 2005.

BOULARBAH, A. et al. Heavy metal contamination from mining sites in South Morocco: 2. Assessmant of metal accumulation and toxicity in plants. Chemosphere, v.63, n.5, p.811-817, 2006.

CAO, X.; MA, L.Q.; TU, C. Antioxidative responses to arsenic in the arsenichyperaccumulator Chinese brake fern (Pteris vittata L.). Environmental Pollution, v.128, n.3, p.317-325, 2004. 
CARBONELL-BARRACHINA, A. A. et al. The influence of arsenite concentration on arsenic accumulation im tomato and bean plants. Scientia Horticulturae, v.71, n.1, p.167-176, 1997.

CARLBERG, C.; MANNERVIK, B. Glutathione reductase. Methods in Enzymology, v.113, p.488-495, 1985.

CHANCE, B.; MAEHLEY, A. C. Assay of catalases and peroxidases. Methods Enzymology, v. 2, p. 764-775, 1955.

CLARK, R. B. Characterization of phosphatase of intact maize roots. Journal Agricultural Food Chemistry, v.23, n.3, p.458-460, 1975.

FRENCH, C. J.; DICKINSON, N. M.; PUTWAIN, P. D. Woody biomass phytoremediation of contaminated brownfield land. Environmental Pollution, v.141, n.3, p.387-395, 2006.

GIANNOPOLITIS, C. N.; RIES, S. K. Superoxide dismutases. Plant Physiology, v.59, n.2, p.309-314, 1977.

HAVIR, E. A.; MCHALE, N. A.Biochemical and developmental characterization of multiple forms of catalase in tobacco leaves. Plant

Physiology, v.84, p.450-455, 1987.

HUNT, R. Plant growth analysis. London, Edward Arnold, 1978.

ISRAR, M. et al. Bioaccumulation and physiological effects of mercury in Sesbania drummondii. Chemosphere, v.65, n.4, p.591598, 2006.

KAR, M.; MISHRA, D. Catalase, peroxidase, and polyphenoloxidase activities during rice leaf senencence. Plant Physiology, v.57, n.2, p.315-319, 1976.

KOSHIBA, T. Cytosolic ascorbate peroxidase in seedlings and leaves of maize (Zea mays). Plant Cell Physiology, v.34, n.5, p.713-721, 1993.

LINS, C. E. L. et al. Growth of mycorrhized seedlings of Leucaena leucocephala (Lam.) de Wit. in a copper contaminated soil. Applied Soil Ecology, v.31, n.3, p.181-185, 2006.
MADHAVA RAO, K. V.; SRESTY, T. V. S. Antioxidative parameters in the seedlings of pigeonpea (Cajanus cajan (L.) Millspaugh) in response to $\mathrm{Zn}$ e Ni stresses. Plant Science, v.157, n.1, p.113-128, 2000.

MADEJÓN, P.; LEPP, N. W. Arsenic in soils and plants of woodland regenerated on an arseniccontaminated substrate: A sustainable natural remediation? Science of the Total Environment, v.379, n.2, p.256-262, 2007.

MASCHER, R. et al. Arsenate toxicity: effects on oxidative stress response molecules and enzymes in red clover plants. Plant Science, v.163, n.5, p.961-969, 2002.

MEHARG, A. A.; HARTLEY-WHITAKER, J. Arsenic uptake and metabolism in arsenic resistant and nonresistant plant species. New Phytologist, v.154, n.1, p.29-43, 2002.

MITTLER, R. Oxidative stress, antioxidants and stress tolerance. Trends in Plant Science, v.7, n.9, p.405-410, 2002.

MITEVA, E. et al. Arsenic as a factor affectin virus infection in tomato plants: changes in plant growth, peroxidase activity and chloroplast pigments. Scientia Horticulturae, v.105, n.3, p.343-358, 2005.

MYLONA, P. V.; POLIDOROS, A. N.; SCANDALIOS, J. G. Modulation of antioxidant responses by arsenic in maize. Free Radical Biology \&

Medicine, v.25, n.4, p.576-585, 1998.

NAKANO, Y.; ASADA, K. Hydrogen peroxide is sacavenged by ascorbate-specific peroxidase in spinach chloroplasts. Plant Cell Physiology, v.22, n.5, p.867-880, 1981.

PAIVOKE, A. E. A.; SIMOLA, L. K. Arsenate toxicity to Pisum sativum: mineral nutrients, chorophyll content, and phytase activity. Ecotoxicology and Environmental Safety, v.49, n.2, p.111-121, 2001.

PEIXOTO, P. H. P. et al. Aluminum effects on lipid peroxidation and on activies of enzymes of oxidative metabolism in sorghum. Revista Brasileira de Fisiologia Vegetal, v.11, n.3, p.137-143, 1999.

R. Árvore, Viçosa-MG, v.33, n.2, p.245-254, 2009 
RONSEIN, G. E. et al. Oxidação de proteínas por oxigênio singlete: mecanismos de dano, estratégias para detecção e implicações biológicas. Química Nova, v.29, n.3, p.563$568,2006$.

ROSEN, B. P. Biochemistry of arsenic detoxification. FEBS Letters, v.529, n.1, p.86-92, 2002.

SAHI, S. V. et al. Accumulation, speciation and cellular localization of copper in Sesbania drummondii. Chemosphere, v.67, NUMERO, p.2257-2266, 2007.

SANTOS, G. A. Crescimento e respostas antioxidantes de macrófitas aquáticas submetidas ao arsênio. 2006. 45f. Dissertação (Mestrado emFisiologia Vegetal) - Universidade Federal de Viçosa, Viçosa, MG, 2006.
SARCINELLI, T.S. et al. Sintomas de deficiência nutricional em mudas de Acacia holosericea em resposta à omissão de macronutrientes. Revista Árvore, v.28, n.2, p.173-181, 2004.

SINGH, N. et al. Metabolic adaptations to arsenicinduced oxidative stress in Pteris vittata $\mathrm{L}$. and Pteris ensiformis L. Plant Science, v.170, n.2, p.274-282, 2006.

TAPIO, S.; GROSCHE, B. Arsenic in the aetiology of cancer. Mutation Research, v.612, n.3, p.215-246, 2006.

WONG, M. H. Ecological restoration of mine degraded soils, with emphasis on metal contaminated soils. Chemosphere, v.50, n.6, p.775-780, 2003.

ZHANG, Z.Q. et al. Soil seed blank as an input of seed source in revegetation of lead/zinc mine tailings.

Restoration Ecology, v.9, n.4, p.378-385, 2001. 